Revue d'histoire de l'Amérique française

REVUE D.HISTOIRE DE L'AMÉRIQUE FRANÇAISE

\title{
Les obstacles sur la voie du sacerdoce chez les Inuits du Canada. Manernaluk, Sikkuaq et les Oblats
}

\section{Frédéric Benjamin Laugrand}

Volume 72, numéro 1, été 2018

URI : https://id.erudit.org/iderudit/1051146ar

DOI : https://doi.org/10.7202/1051146ar

Aller au sommaire du numéro

Éditeur(s)

Institut d'histoire de l'Amérique française

ISSN

0035-2357 (imprimé)

1492-1383 (numérique)

Découvrir la revue

Citer cet article

Laugrand, F. B. (2018). Les obstacles sur la voie du sacerdoce chez les Inuits du Canada. Manernaluk, Sikkuaq et les Oblats. Revue d'histoire de l'Amérique française, 72(1), 53-80. https://doi.org/10.7202/1051146ar
Résumé de l'article

Au Canada, les Oblats de Marie-Immaculée ont participé intensivement à l'évangélisation des populations autochtones. Historiens et anthropologues ont analysé cette histoire complexe et mis l'accent sur les stratégies de ces missionnaires pour la plupart issus de France, de Belgique et d'autres pays européens. Ils ont cependant laissé de côté l'étude des vocations féminines et masculines autochtones. Cet article examine deux trajectoires masculines, celle d'Anthony Manernaluk et celle de Nick Sikkuaq, tout deux frères de sang et frères coadjuteurs. L'auteur montre que le célibat comme les exigences sociales des Oblats étaient difficilement acceptables et des obstacles pour le sacerdoce, les Inuits demeurant attachés à d'autres sociabilités. 


\title{
Les obstacles sur la voie du sacerdoce chez les Inuits du Canada. Manernaluk, Sikkuaq et les Oblats
}

\author{
Frédéric BenJamin LaUgrand
}

Université Laval

RÉsumÉ • Au Canada, les Oblats de Marie-Immaculée ont participé intensivement à l'évangélisation des populations autochtones. Historiens et anthropologues ont analysé cette histoire complexe et mis l'accent sur les stratégies de ces missionnaires pour la plupart issus de France, de Belgique et d'autres pays européens. Ils ont cependant laissé de côté l'étude des vocations féminines et masculines autochtones. Cet article examine deux trajectoires masculines, celle d'Anthony Manernaluk et celle de Nick Sikkuaq, tout deux frères de sang et frères coadjuteurs. L'auteur montre que le célibat comme les exigences sociales des Oblats étaient difficilement acceptables et des obstacles pour le sacerdoce, les Inuits demeurant attachés à d'autres sociabilités.

ABstract - In Canada, the Oblates of Mary Immaculate were actively invested in the evangelization of Indigenous peoples. Historians and anthropologists have analyzed this complex history, focusing on the strategies used by missionaries who, for the most part, originated from France, Belgium and other European countries. However, there has been no study of the vocations of Indigenous women and men. This paper deals with two male trajectories, that of Anthony Manernaluk and Nick Sikkuaq, who were both blood brothers and coadjutor brothers. The author shows that celibacy as well as the social requirements of the Oblates were challenging demands and constituted obstacles to priesthood for the Inuits who had been brought up with other sets of sociability markers. 


\section{INTRODUCTION'}

En 1841, l'arrivée des missionnaires oblats, à la suite de l'appel de $\mathrm{M}^{\text {gr }}$ Ignace Bourget, ouvre une nouvelle période d'évangélisation des peuples autochtones du Canada. En provenance d'Europe, de nombreux missionnaires furent envoyés dans plusieurs régions, d'abord dans l'ouest et l'est du pays, puis dans le Grand Nord, sur les rives du Mackenzie dès 1849 et dans l'Arctique de l'Est, à partir de 1912. Un siècle plus tard, ces missionnaires peuvent se réjouir d'avoir réalisé un grand nombre de conversions et christianisé des cultures fort différentes. Ils doivent cependant aussi constater leur échec sur le plan des vocations indigènes, seuls quelques rares Autochtones ayant décidé de devenir prêtres. Envoyées à la même époque pour aider les Oblats à soigner et à éduquer leurs nouvelles ouailles, les congrégations féminines remportèrent plus de succès dans la mesure où l'on estime aujourd'hui à plus d'une soixantaine le nombre de femmes autochtones ayant pris l'habit. Comment, donc, expliquer ce maigre succès des Oblats auprès des hommes, alors que leurs contemporains, les missionnaires anglicans, ont su recruter et former des centaines de pasteurs $^{2}$ ?

Parions qu'en examinant les deux seuls cas connus à ce jour, à savoir celui d'Antony Manernaluk et de Nick Sikkuaq, qui ont eu momentanément une vocation et reçu une formation pour devenir des frères coadjuteurs, nous parviendrons à éclairer les raisons de cet absence de prêtres inuits chez les Oblats ${ }^{3}$. Nous verrons que les archives restent parfois muettes, de sorte qu'il nous faudra demeurer prudent dans les hypothèses formulées.

Sur le terrain, les missionnaires oblats ont déployé des stratégies d'adaptation, n'hésitant pas à apprendre une multitude de langues autochtones et à vivre comme des nomades. Dans la masse considérable de textes et d'images qu'ils laissent à la postérité, leur volonté de stimuler l’intérêt pour les missions et de susciter de nouvelles vocations paraît une évidence, d'autant plus que leurs actions se sont déroulées dans un contexte de rivalités religieuses.

1. J'exprime ma gratitude au père Robert Lechat (o.m.i.) ainsi qu'aux deux évaluateurs anonymes pour leurs remarques qui ont permis de bonifier ce texte. Je remercie aussi l'Université catholique de Louvain (UCL) et le Fonds de la Recherche Scientifique-FNRS (Mandat Ulysse) pour sa subvention $\mathrm{n}^{\circ}$ (F.6002.17).

2. Christopher Trott, "Mission and Opposition in North Baffin Island», Journal of the Canadian Church Historical Society, 30 (1998), p. 31-55.

3. L’ordre des Oblats de Marie Immaculée a été fondé en 1816 par Eugène de Mazenod, archevêque de Marseille. Il plonge ses racines dans le catholicisme ultramontain du XIX siècle. 
Contrairement aux Anglicans au XX $\mathrm{X}^{\mathrm{e}}$ siècle, les Oblats ne souhaitaient pas former immédiatement un clergé indigène. Ils espéraient plutôt assurer un renouvellement des effectifs, comme si cette manne missionnaire était garantie à tout jamais. Mais dans les années 1950, à mesure que les succès du christianisme se font de plus en plus visibles, les vocations indiennes et esquimaudes deviennent une préoccupation.

Dans l'Arctique, les Oblats ont longtemps voulu s'assurer du contrôle total de l'évangélisation et de la diffusion des textes bibliques dont ils craignaient les interprétations littérales. Dans les missions du Nord, en effet, certains chamanes et chefs de camps s'étaient déjà emparés du message biblique et agissaient en prophètes. Plusieurs avaient même inventé leur propre religion en proposant des synthèses inédites du chamanisme et du christianisme. Dans les années 1940, dans la région de Baker Lake, le cas de Sulutnar (Suluk) avait laissé des séquelles. Le père Henri-Paul Dionne, par exemple, décrivait ce personnage comme «un visionnaire éclectique» et un "illuminé» donnant dans la superstition, son mouvement n'offrant qu' "un mélange de sorcellerie et de protestantisme ". Le missionnaire s'indignait de le voir utiliser des croix à mauvais escient et d'entendre de ses ouailles qu'il faisait apparaître les esprits des défunts, qu'il organisait des confessions avec son chien et encourageait ses adeptes à échanger leurs femmes.

Au milieu des années 1940, la situation semblait toutefois sur le point de basculer dans le Vicariat de la Baie d'Hudson. À Chesterfield Inlet, où les Oblats avaient établi leur toute première mission de l'Arctique de l'Est et où les Sœurs grises étaient arrivées en renfort en 1931 pour s'occuper d'un hôpital et d'une école, les derniers chamanes, comme Paapak, Qimuksiraaq et Talleriktok, s'étaient convertis ${ }^{6}$. En 1944, l'espoir renaissait du fait qu'une première femme, Mélanie Arnaluk, malheureusement décédée peu après, avait exprimé le vœu de devenir une sœur missionnaire. En 1948, une seconde femme, Pélagie Inuk, avait à son tour été admise comme postulante au grand désespoir de sa famille de chamanes ${ }^{7}$. En somme, le chamanisme semblait partout reculer et le christianisme triompher. En 1951, sœur Pélagie Inuk prononçait ses premiers vœux et

4. Charles Choque, Joseph Buliard, pêcheur d'hommes: de la Franche-Comté au Grand Nord canadien (1914-1956) (Longueuil, Le Préambule, 1985), p. 117-118.

5. C. Choque, Joseph Buliard..., p. 117-118.

6. Frédéric Laugrand, Mourir et renaître. La réception du christianisme par les Inuit de l'Arctique de l'Est canadien (Québec, Les Presses de l’Université Laval, 2002), p. 168.

7. Frédéric Laugrand et Jarich Oosten, «The Case of Pelagie Inuk: The Only Inuk Woman to Become a Grey Nun », Études Inuit Studies, 38, 1-2 (2014), p. 157-176. 
plusieurs autres candidates étaient en lice. Célébrée en grande pompe, la cérémonie de prise de voile de Pélagie fut soigneusement filmée par $l^{\prime}$ Office national du film $(\mathrm{ONF})^{8}$. De nombreux articles de presse au Canada et dans le monde firent écho à cette ordination d'une première sœur esquimaude. Une première fleur, disait-on, avait émergé des glaces ${ }^{9}$.

Du côté des hommes, deux frères allaient bientôt s'engager dans une vocation religieuse: Anthony Manernaluk et Nick Sikkuaq. Pour les Oblats, les terres stériles du Kivalliq devenaient maintenant fertiles, d'autant plus que plusieurs catéchistes, comme Jean Ayaruaq à Chesterfield Inlet, agissaient déjà comme des fers de lance du catholicisme. Et pourtant, les deux vocations indigènes qui suscitaient tant de joie allaient faire long feu. Plus que cela, elles ne tardèrent pas à se révéler des échecs cuisants pour les Oblats qui devront, au milieu des années 1970, repenser leur stratégie de formation d'une relève indigène.

Dans cet article, je propose de revenir sur cette période historique des années 1950-1960 afin d'interroger les raisons de l'échec des Oblats en examinant les deux uniques cas de vocations masculines jamais documentées pour les Inuits, à savoir celles des frères Anthony Manernaluk et Nick Sikkuaq. Je laisse de côté une autre vocation qui n’a pas duré plus longtemps et qui concerne le Groenland où, en 1964 et dans un tout autre contexte, le père Finn Lynge est devenu, pendant quelques mois, le premier et unique "prêtre oblat esquimau». Je n'aborderai pas davantage ici les vocations féminines, les cas de la sœur Pélagie Inuk et de plusieurs autres postulantes - Blandine Nennout, Alexina Nutaradjuk et Salome Paoktut - ayant déjà été analysés ${ }^{10}$. À cet égard, pour comprendre la rareté des vocations, il ne suffit pas d'évoquer "la mentalité paternaliste et civilisatrice des premiers missionnaires ${ }^{11}$ ", il faut voir aussi ce qui, du point de vue des Inuits, de leurs mentalités et modes de vie, a brutalement arrêté les premières vocations sachant, par ailleurs, que dès l'introduction du christianisme, les Inuits ont eu un réel engouement pour cette religion ${ }^{12}$.

8. Gontran Laviolette, «La première religieuse du pays des glaces », La liberté et le patriote, Winnipeg, 1951, p. 2.

9. Voir par exemple, Charles Choque, «Sœur Pélagie Pubvalerak, première religieuse esquimaude», Pôle et tropiques, 6 (1951) p. 2-5 ; Adrien Bouffard, «Une fleur des neiges: Naya Pélagie», Eskimo (1956), p. 99-106.

10. F. Laugrand et J. Oosten, "The case of Pelagie Inuk... », p. 157-176.

11. Anny Morissette, «La culture oblate et la religion invisible dans les missions amérindiennes du Québec", dans Marie-Pierre Bousquet et Robert Crépeau, dir., Dynamiques religieuses des autochtones des Amériques (Paris, Karthala, 2009), p. 290.

12. En témoigne ces rites de conversion collective comme le siqqitiq analysés dans F. Laugrand, Mourir et renaître..., p. 449-472. 
Qui étaient donc Anthony Manernaluk et Nick Sikkuaq? Pourquoi ont-ils été tentés par une vocation religieuse au contact des missionnaires oblats? Quel a été leur cheminement dans cette formation sacerdotale? Enfin, comment expliquer cet échec des Oblats dans le recrutement de prêtres inuits à une époque où le christianisme semble triompher et où le Nord fait l'objet d'une occidentalisation sous l'effet combiné de la sédentarisation, de la scolarisation des Inuits et de la modernisation des infrastructures? Quels obstacles culturels expliquent cette impasse dans laquelle se trouvent les missionnaires oblats?

La réflexion se fera en trois temps. Il s'agira d'abord de décrire et d'analyser chacune des deux trajectoires à partir de documents conservés dans les archives. Les informations sont extraites du Codex historicus de la mission de Baker Lake, de rapports inédits, de la correspondance missionnaire et de divers articles de revues missionnaires qui servaient d'outils de propagande. Les Oblats espéraient stimuler l'intérêt du grand public et des membres bienfaiteurs des missions, mais ils visaient aussi à recruter de nouvelles vocations. Le corpus comprend enfin quelques témoignages oraux recueillis lors de mes recherches sur la réception du christianisme en milieu inuit ${ }^{13}$.

\section{DEUX FRÈRES DOUBLEMENT FRÈRES: MANERNALUK ET SIKKUAQ}

Selon les informations consignées dans le Codex historicus de la mission catholique de Garry Lake, les frères Anthony Manernaluk et Nicolas Sikkuaq ont été tous les deux convertis à la fin des années 1940 par le père Joseph Buliard, chargé à l'époque de la desserte de N.D. du T.S. Rosaire, à Garry Lake, T.N.O. ${ }^{14}$.

Le père Joseph Buliard, auquel le père Charles Choque a consacré une biographie $^{15}$, est né en 1914 en Franche-Comté. Son séjour nordique est émaillé de deux drames. En 1939, il tombe d'abord accidentellement dans de l'eau glaciale et développe la gangrène. Les Oblats organisent son évacuation d'urgence par avion. Le missionnaire reprend du service et voyage avec des Inuits dans la région de Repulse Bay, de la rivière Back et de Baker Lake. En 1949, il est envoyé à la mission de Garry Lake où il est accueilli par des familles catholiques, mais raillé par d'autres qui adhèrent

13. Voir, par exemple, Frédéric Laugrand et Jarich Oosten, Inuit Shamanism and Christianity. Transitions and Transformations in the Twentieth Century (Montréal, McGill-Queen's University Press, 2010).

14. Charles Choque, «Histoire de la Mission catholique de Baker Lake, 1927-1965 » (2011), manuscrit inédit transmis à l'auteur, p. 35 .

15. C. Choque, Joseph Buliard... 
au protestantisme. À ce titre, Buliard écrit : «il est plus facile de convertir un vieux sorcier qu'un protestant orgueilleux ${ }^{16}$ ». Le missionnaire sillonne le territoire, parfois en traîneau à chiens sur des distances de $2000 \mathrm{~km}$ avec ses guides. Il découvre la dure réalité des famines qui ravagent les familles. Puis, en 1956, il disparaît mystérieusement dans la toundra, vraisemblablement mort noyé ${ }^{17}$.

Manernaluk et Sikkuaq étaient donc des frères au sens biologique et religieux du terme. Ils avaient été adoptés par le père Buliard qui, après le décès brutal de leurs parents, les avait pris sous son aile, suivant là le système d'adoption des Inuits. Manernaluk deviendra frère oblat en 1958 et Sikkuaq en 1961. Leur trajectoire religieuse se termine cependant très vite, puisqu'en 1962 Manernaluk quitte l'ordre des Oblats et Sikkuaq le fera deux ans plus tard.

\section{ANTHONY MANERNALUK}

Manernaluk est né le 4 avril 1937 sur la Perry River. Il a été baptisé par le père Joseph Buliard, le 3 ou le 4 avril 1948, sous le nom d'Antoine, au camp de Kuksut, un chamane qui s'est longtemps opposé au missionnaire. Les deux frères cadets de Manernaluk furent baptisés en même temps et adoptés, eux, par le père Henry à Gjoa Haven. Anarkrarnerk prit le nom de Louis, et Sikkuaq celui de Nicolas. Anthony Manernaluk fit sa première communion le jour de son baptême à l'âge de 11 ans. Sa confirmation par $\mathrm{M}^{\mathrm{gr}}$ M. Lacroix a lieu dix ans plus tard, le 29 mars 1958, à Baker Lake.

Selon les informations recueillies dans les archives oblates, les parents de Manernaluk, Vital Awingark et Elizabeth Kringor, moururent prématurément de l'influenza le 30 juin et le 21 juillet 1952. Dans son journal, le père Joseph Buliard décrit ce couple comme appartenant certainement «à (s)es meilleurs catholiques, toujours très bons et très fidèles, élevant bien leurs enfants», ajoutant n'avoir "aucune inquiétude sur leur salut», considérant au contraire ces âmes "simples et droites" ${ }^{18}$.

Selon l'expression du père Thibert, Manernaluk se serait littéralement «donné» au père Buliard le 9 mai 1953 lorsqu'il a accepté, à l'âge de 16 ans, de le servir comme ikajuqti, guide de voyage. Charles Choque précise: "À partir de ce moment, on le considère comme donné au Père Buliard ${ }^{19}$."

16. C. Choque, Joseph Buliard..., p. 192.

17. C. Choque, Joseph Buliard..., p. 192.

18. Archives Deschâtelets (AD), HH4230 A62C 120, Frère coadjuteur Oblat esquimau. Le R.F. Anthony Manernaluk, O.M.I.

19. AD, HH-4865-A62C-29, ex.1. 
Le père Choque explique que Buliard a souvent essayé de mobiliser des jeunes et de les éduquer, convaincu qu'ils ont «le cœur généreux et fort» et sont «capables de tenir tête au premier sorcier ou à la première crapule $^{20}$ ». Rappelons également qu'après son grave accident en 1939 où il eut les mains gelées, Buliard avait perdu en partie la vue et sa dextérité, ce qui le handicapait considérablement pour missionner dans la toundra. Quant aux Inuits, ils estimaient que Manernaluk était bien l'adopté du père Buliard ${ }^{21}$.

\section{Manernaluk, l'adopté du père Joseph Buliard}

Le père Buliard, qui a passé quatre ans en compagnie de Manernaluk, relate leur complicité et comment le jeune Inuk est vite devenu, pour lui, "un petit frère convers sans les vœux»:

Avec Anthony, ma vie a changé un peu. C’est comme si j'avais un frère avec moi, et, peu à peu, je le fais coopérer, non seulement pour le matériel, mais aussi pour les âmes. Il est encore trop jeune pour qu'il prenne une décision. [...] L'ennuyeux c'est que j'ai peur pour sa santé. Évidemment je dois le nourrir, le vêtir et le chausser, mais par son travail, car il est capable tout autant que n'importe quel homme (sauf la force et l'endurance) de chasser, pêcher, faire des filets et m'aider, il paye bien cela. Et en hiver, quand il saura le chemin, car il sait bâtir l'iglou et s'occuper d'une traîne, nous pourrons aller visiter les camps les plus proches et rester tant que nous voudrons, car pas de famille pour nous attendre ${ }^{22}$.

Dès cette époque, Buliard ressent l'intérêt du jeune garçon pour les questions religieuses. Le missionnaire se préoccupe toutefois de sa santé fragile. Plus tard, il renchérit sur les qualités de son aide, déclarant que Manernaluk est un homme intelligent, vaillant et sans reproche, "précieux, pieux et dévoué », et qu'il sera son "unique guide »"

Beaucoup plus tard, le journaliste David Pelly, qui a interviewé Anthony Manernaluk et ses deux frères sur la vie à la mission de Garry Lake, fait état de cette profonde amitié qui unissait Manernaluk au père Buliard. Pelly décrit l'Inuk comme un excellent guide du missionnaire, alors que Buliard, lui, laisse entendre qu'il devait former son aide, certes très débrouillard, mais encore craintif de voyager seul et un peu lent à construire l'iglou ou raboter le traineau. Pelly rapporte qu'après tant de

20. C. Choque, Joseph Buliard..., p. 214.

21. C. Choque, "Histoire de la Mission catholique de Baker Lake... », p. 58.

22. AD, HH4230 A62C 120, Frère coadjuteur...

23. AD, HH4230 A62C 120, Frère coadjuteur... 
voyages effectués ensemble, Manernaluk se souvenait du père Buliard avec fierté et que les deux hommes se divisaient les tâches comme un couple: «Manernaluk parlait de Buliard comme d'un père, et même plus. "Jadis, il avait toujours froid aux mains et aux pieds", se souvenait Manernaluk [...] "e Mais quand je voyageais avec lui, il n'avait jamais froid", ajouta-t-il. [...] Et le temps que Manernaluk se glisse dans l'iglou, "le père Buliard avait déjà préparé le thé et la bannique"24."

L'amitié des deux hommes fut cependant de courte durée. À l'été 1956, Manernaluk dut abandonner son compagnon pour des problèmes de santé. Diagnostiqué tuberculeux, il fut envoyé au sanatorium de Brandon, au Manitoba. Manernaluk confia à Pelly qu'il fit tout en son pouvoir pour rester, souhaitant âprement demeurer aux côtés du père Buliard mais qu'on ne lui laissa pas le choix. Manernaluk fut hospitalisé du 10 juillet 1956 au 30 août $1957^{25}$. Buliard dut alors chercher un autre guide mais le 24 octobre 1956, alors qu'il partit seul relever ses filets de pêche, il disparut définitivement dans le blizzard.

Un missionnaire anglican répandit la rumeur d'un assassinat du père Buliard par des Inuits, mais l'enquête ne révéla rien. Au contraire, les Inuits de la région furent profondément attristés par cette disparition. Pelly rapporte la réaction de Manernaluk à cette nouvelle: «Lorsque j’ai entendu que le père Buliard était porté disparu, j'ai eu le sentiment de perdre un parent ${ }^{26}$.» À Garry Lake, personne ne remplaça le père Buliard et moins de deux ans plus tard, tous les Inuits de Hanningajuq périrent de la famine, ou furent évacués à Baker Lake ${ }^{27}$.

Selon les Oblats, la vocation de Manernaluk aurait émergé lors de son séjour à l'hôpital, en 1956-1957, au moment où il apprit le décès du père Buliard. En mars 1958, peu de temps après sa confirmation, Manernaluk est envoyé à Gjoa Haven par $\mathrm{M}^{\mathrm{gr}}$ Lacroix, l'évêque du Vicariat de la Baie d'Hudson, afin de commencer son postulat avec le père P. Henry. Plusieurs journaux de l'époque, comme le Indian Record, font état de cette première vocation masculine inuite au Canada ${ }^{28}$. Les Oblats étaient comblés par la nouvelle et, dès 1960, un article du journal L'Apostolat annonçait triom-

24. David Pelly, Ukkusiksalik, The People's Story (Toronto, Dundurn Press, 2015), p. 3048. Les traductions sont de l'auteur.

25. Selon les archives, Manernaluk retourna ensuite à la mission de Garry Lake pendant une période de six mois, le père Ernest Trinel ayant alors remplacé le père Buliard. AD, HH-4865-A62C-29, ex.1.

26. C. Choque, Joseph Buliard...

27. Selon les archives, entre 1955 et 1957, les Inuits de cette région ont connu de sévères famines. Après le décès de 16 d'entre eux, le gouvernement fédéral organisa en 1957-1958 l'évacuation des survivants vers le village de Baker Lake. AD, HH4230 A62C 120, Frère coadjuteur...

28. Anonyme, «First Eskimo Lay Brother Takes Vows», Indian Record, 21 (avril 1958), p. 2. 
phalement: "Anthony Manernaluk Esquimau à 100\%. Après Sœur Pélagie [...], voici Frère Anthony, le premier oblat esquimau ${ }^{29}{ }^{\prime}$.

Le père Henry se disait très impressionné par le jeune Inuk, au point de recommander à ses confrères de prendre en compte toute son expérience. Il écrit,

Le temps qu'Anthony passa avec moi devrait lui compter comme postulat. Il donna pleine satisfaction. Je crois que c'est une belle acquisition pour la Congrégation et la conversion de ceux de sa race. Il est zélé d’un zèle intelligent, fait de patience, de renoncement et d'humilité. S'il continue dans la voix où il marche actuellement, il sera un précieux compagnon pour n'importe quel père, soit chez les Esquimaux, soit même ailleurs ${ }^{30}$.

Ces commentaires laissent entendre le dévouement du jeune homme, considéré comme une excellente recrue.

Le 2 septembre 1958, Manernaluk quitte donc Gjoa Haven pour rejoindre le noviciat des Oblats à Saint-Norbert, au Manitoba, où il arrive le 6 septembre 1958. Il y prend l'habit de frère le 7 décembre 1958. Son père religieux, soit la personne qui est responsable de lui selon les usages en la matière, est le père Beauregard, assisté par le père E. Daniélo déjà très familier des Inuits auprès desquels il a été missionnaire.

Manernaluk prononce ses premiers vœux le 8 décembre 1959 devant une dizaine de patients inuits convalescents des sanatoriums de Ninette, Brandon, Saint-Vital et Winnipeg et en présence des pères M. Rio, E. Daniélo et R. Beauregard; ce dernier préside la cérémonie. Il passe ensuite quelques mois à Saint-Norbert, visite Saint-Boniface et Saint-Vital. Le 16 décembre 1959, il écrit au père Thibert toute sa satisfaction d'avoir pu prononcer ses premiers vœux. En 1960, il reçoit sa première obédience pour Gjoa Haven où il aide les pères P. Henry, P. Goussaert et J. Vermeersch. Ses seconds et troisième vœux sont prononcés dans ce village les 8 décembre 1960 et 1961 respectivement. À la même époque, Manernaluk séjourne également à Pelly Bay auprès du père Van de Velde qui, en plus de lui confier du travail manuel, lui fait donner des leçons d'inuktitut au père Jean-Guy Roberge, une nouvelle recrue ${ }^{31}$. À la fin de l’année 1961, Manernaluk reçoit sa seconde obédience, cette fois pour

29. Anonyme, «Premières vocations esquimaudes. Anthony Manernaluk», L'Apostolat (février 1960), p. 18.

30. AD, HH4230 A62C 120, Frère coadjuteur...

31. Sur cette mission catholique, voir Jarich Oosten et Cornelius Remie, "The Birth of a Catholic Inuit Community. The Transition to Christianity in Pelly Bay, Nunavut, 1935-1950 ", Études Inuit Studies, 26, 1 (2002), p. 109-141. 
Baker Lake où il se rend aux côtés du père Charles Choque, alors Supérieur oblat.

Les Oblats et la communauté catholique se montrent très heureux de ces développements et entre 1960 et 1962, la presse oblate multiplie les articles sur son travail et son parcours, tout en célébrant sa nomination et celle de son frère, Nick Sikkuaq, qui vient de le rejoindre. Manernaluk apparaît même sur les écrans de télévision à Winnipeg, interviewé par un autre missionnaire, vétéran de l'Arctique, le père Rio et un journaliste ${ }^{32}$. À l'époque, Manernaluk parle couramment l'anglais et le français et il a réussi tous ses examens. Les Oblats soulignent son aide précieuse pour la rédaction de certains documents et ses compétences comme travailleur manuel, en indiquant qu'il a construit un grenier, un garage et une maison pour les personnes âgées ${ }^{33}$. Cette polyvalence en fait un missionnaire indigène très prometteur.

\section{L'engagement d'Anthony Manernaluk}

Le journal L'Apostolat publie plusieurs lettres et extraits de lettres qu'Anthony Manernaluk a écrites au père Arthur Thibert qu'il aidait dans sa traduction du Missel ${ }^{13}$. Les passages suivants sont tirés de cette correspondance. Ces extraits éclairent la manière dont Manernaluk saisit le christianisme.

Un premier extrait, d'une lettre datée de janvier 1958, montre que l'intérêt de Manernaluk pour le christianisme se fonde au départ sur sa conviction que les Inuits vivent dans des conditions difficiles et qu'ils ont besoin d'aide, un point de vue qu'il tient peut-être des Oblats:

C’est Anthony qui écrit. Je t'envoie une petite page intitulée «Prions Dieu, notre Père, car nous manquons de tout». Si tu juges la chose opportune, je crois que ça pourrait être utile à mes compatriotes. Ce sont mes propres réflexions qui m’ont porté à écrire cela, car les Esquimaux sont dans une grande misère.

Prions pour recevoir la vie de notre âme et de notre corps, de la part du Bon Dieu.

Notre père qui es au ciel, tu es le Créateur tout-puissant: c'est Toi qui as tout fait, partout sur la terre: tous les animaux et tous les hommes sont à toi.

32. AD, HH4230 A62C 120, Frère coadjuteur...

33. AD, HH4230 A62C 120, Frère coadjuteur...

34. Anonyme, "Premières vocations esquimaudes... ", L'Apostolat (février 1960), p. 18. 
Je veux me consacrer tout entier à Toi: prends-moi avec tout ce que j'ai. Je veux accomplir Ta volonté même si parfois cela semble me déplaire, comme la maladie et la misère. D’avance, je veux Te dire: Que Ta sainte volonté soit faite ${ }^{35}$ !

Les animaux, à la fois proies et partenaires des Inuits, sont mentionnés par le frère qui semble considérer Dieu comme un grand esprit (inua), un créateur et maître du gibier. En cela il demeure très proche des conceptions chamaniques.

Sur le plan spirituel, les sources d'inspiration de Manernaluk apparaissent dans une seconde lettre, alors qu'il vit encore à Baker Lake, en mars 1958:

Merci pour les écrits de sainte Thérèse de l'Enfant-Jésus, en Esquimau, que j'ai reçus de ta part. Cette vie m'a beaucoup réjoui: rien qu'à lire cela, ça nous fait désirer d'être meilleur. Je comprends de mieux en mieux la beauté de la vie chrétienne. J'en lis des passages tous les jours. Je suis rendu à la page 124 et je vais tout lire, absolument tout.

Tu me demandes ce que je fais à Baker Lake. Tous les jours je prie beaucoup aux intentions des prêtres, des frères et des religieuses. Je prie beaucoup aussi pour les Esquimaux qui n’ont pas encore la foi et qui hésitent à suivre Jésus...

Il y aura bientôt trois ou quatre années que je travaille pour les prêtres. Actuellement je suis sacristain, cuisinier, et je fais manger nos chiens ${ }^{36}$.

Cette lettre témoigne de la piété de Manernaluk qui a intégré la dévotion à sainte Thérèse, une figure introduite jadis par $\mathrm{M}^{\mathrm{gr}} \mathrm{A}$. Turquetil. Manernaluk consacre beaucoup de temps à la prière et à diverses tâches domestiques. Il s'identifie dorénavant aussi comme membre à part entière du personnel missionnaire et souscrit à l'objectif de convertir les Inuits.

La troisième lettre, datée aussi de mai 1958, revient sur l'émergence de sa vocation. Il apparaît que Manernaluk est bien un candidat que le père Buliard a encouragé à devenir frère.

Autrefois le père que j'accompagnais en voyage, le père Joseph Buliard, me disait ceci: «Ton avenir est entre tes mains : si tu veux devenir frère oblat, tu le pourras; par contre, si tu veux fonder un foyer, libre à toi.»

Maintenant je suis décidé à donner ma vie à Jésus : que toutes mes activités soient pour lui et pour ses missionnaires qui, à leur tour, consacrent tout leur vie pour les Esquimaux.

35. Anonyme, "Premières vocations esquimaudes... ", Lettre de janvier 1958.

36. Anonyme, " Premières vocations esquimaudes... ", Lettre de mars 1958. 
Je suis prêt à renoncer à fonder un foyer et à posséder quoi que ce soit personnellement. Ainsi je serai libre de devenir frère coadjuteur. Mon plus grand désir est de tout faire par obéissance et cela pour toute ma vie ${ }^{37}$.

Cette lettre suggère que Manernaluk a bien identifié les exigences de sa vocation, notamment le renoncement au mariage et à la famille. Nous y reviendrons.

Les deux autres lettres, datées de septembre 1958 et mars 1959 respectivement, laissent entendre qu'au noviciat, Manernaluk fut amené à faire une multitude d'activités, et pas seulement des prières:

C'est donc le 6 septembre que j'arrivai au Noviciat de Saint-Norbert. Depuis lors je me suis mis au travail pour récolter les patates, avoir soin des poules et aider aux divers travaux. La prière et le chapelet sont de tous les jours et je suis très heureux.

Joyeuses Pâques, Père! Alors, nous sommes à la joie! Comme nous étions tristes, en cette semaine sainte. Le vendredi saint on a gardé le silence toute la journée. Maintenant nous pouvons causer et manger de la viande! Nous avons souvent médité sur la mort de Notre-Seigneur et sur sa croix, et maintenant sa résurrection nous prouve aussi son amour pour nous ${ }^{38}$.

Au noviciat de Saint-Norbert, le jeune homme semble s'adapter relativement bien à sa nouvelle vie. Ses activités sont variées et l'on peut aisément concevoir sa joie à sortir du Carême, le régime alimentaire inuit étant basé sur la consommation quotidienne de grandes quantités de viande et de poisson. Comme de nombreux Inuits, Manernaluk est fasciné par La vie des saints transcrite en syllabique par le père E. Daniélo, un livre qu'il lit à partir de ses propres grilles ${ }^{39}$. En juillet et en décembre 1959, il est affecté par la maladie de sa sœur:

Je suis heureux au Noviciat de Saint-Norbert: parfois il fait très chaud! En travaillant toute la journée, je suis tout en sueur et ça me donne le rhume. Mais, actuellement, je suis bien et heureux. En imitant les saints et en gardant sa conscience en paix, la mort ne doit pas sembler pénible. Faisons donc notre possible pour imiter les saints qui ont bien vécu...

Ma sœur Alice est encore malade. Prions qu'elle ne se décourage pas. Elle est malade depuis 10 ans, et maintenant elle vivote sur la terre. Si elle meurt, puisse-t-elle aller au ciel, car elle doit se résonner [sic] et accepter son épreuve ${ }^{40}$.

37. Anonyme, " Premières vocations esquimaudes... ", Lettre de septembre 1958.

38. Anonyme, " Premières vocations esquimaudes... ", Lettre de mars 1959.

39. Anonyme, "Premières vocations esquimaudes... ", Lettre de juillet 1959.

40. Anonyme, "Premières vocations esquimaudes... ", Lettre de décembre 1959. 
Je suis vraiment très heureux d'avoir pu faire mes premiers vœux dans la Congrégation des Oblats de Marie Immaculée.

À Noël, je serai avec toi par ma prière. Je prie pour toi, devant le Tabernacle, et je ne t'oublierai pas à la messe de Minuit. Que le Bon Dieu bénisse ton dévouement ${ }^{41}$.

Manernaluk souffre de la chaleur et de rhume et d'être séparé de sa sœur qu'il ne peut aider. Mais il semble trouver consolation dans sa lecture de la vie des saints et dans les fêtes chrétiennes qui rythment sa nouvelle vie.

En 1960, Manernaluk fait parvenir le texte qu'il a rédigé pour ses vœux au père Thibert qui le publie peu après dans La liberté et le patriote, le 5 février $1960^{42}$. Les archives ont conservé le document original en syllabique et la traduction littérale qu'en livre le père Thibert:

Au nom de Notre Seigneur Jésus Christ, en présence de Dieu Unique et en Trois personnes, en présence de Sainte Marie la Vierge, en présence de tous les anges et de tous les Saints, de plus en présence des prêtres et des frères mes associés, voici que devant toi, Père Marcel Rio, délégué de notre Supérieur Général, je te considère comme le représentant de Dieu envers moi, voici que moi, Anthony Manernaluk, je fais vœu à mon Dieu ainsi: je renonce à faire acte de propriété (pauvreté); je ne prendrai point d'épouse (chasteté); j'obéirai absolument à mon supérieur, pour un an. De même devant Dieu, je jure et fais vœu de persévérer pour un an dans la Congrégation ainsi dénommée: Oblat de Marie Immaculée, qui fut conçue sans le péché original. Ainsi que Dieu me soit en aide! Ainsi soit-i $1^{43}$.

Ces lignes mélangent des formules d'usage et un texte idiosyncratique. Elles rappellent combien le christianisme, avec ses multiples figures, est "un bien curieux monothéisme» selon l'expression de Paul Veyne ${ }^{44}$, une dimension que les Inuits, avec leurs traditions chamaniques, percevaient bien. Les vœux d'obéissance ne posaient guère plus de problème, les traditions inuites étant truffées d’interdits rituels. L'abandon de toute vie familiale allait toutefois s'avérer plus difficile à tenir.

41. Anonyme, "Premières vocations esquimaudes... ", Lettre de septembre 1958.

42. La liberté et la patriote, 46, 43 (février 1960), p. 3. La lettre en caractères syllabiques et sa traduction a également été publiée dans L'Ami du foyer (janvier-février 1960).

43. $\mathrm{AD}, \mathrm{HH}-4230-\mathrm{A} 62 \mathrm{C}-10$, ex.1.

44. Paul Veyne, Quand notre monde est devenu chrétien (Paris, Albin Michel, 2007). Avec un brin de provocation, l'historien préfère qualifier le catholicisme de "polythéisme moniste». 


\section{Le renoncement à la vie de frère oblat}

En 1962, Manernaluk est sur le point de regagner le Nord, se préparant à renouveler ses vœux le 8 décembre, lorsqu'un doute l'envahit. Il en informe immédiatement le père Daniélo qui, sous le choc, écrit à son tour au père Haramburu en décembre 1962 :

Je vous avertis tout au début que cette lettre va vous causer de la peine; et c'est le cœur bien gros que moi-même je vous écris. [...] le frère Anthony, qui devait faire ses vœux de trois ans le 8 décembre, m’a avoué hier matin qu'il doute de sa vocation, et qu'en conséquence il ne pense pas pouvoir renouveler ses vœux. Je soupçonnais la chose depuis avant-hier, quand il est venu me voir à mon retour de Fort Alexandre. Il se plaignait alors, d'une manière réticente, qu'il ne pouvait rien apprendre, qu'il devait être une cause de fatigue pour les prêtres n'ayant pas de sujet de conversation avec eux, et qu'il trouvait de grandes difficultés à méditer, prier ${ }^{45}$.

Le père Daniélo explique qu'il a d'abord essayé de rassurer le candidat en lui indiquant qu'il pouvait se limiter à être un frère coadjuteur et ne pas devenir prêtre, le mettant toutefois en garde contre toute forme de tentation. Mais Manernaluk a autre chose en tête. Et Daniélo de poursuivre,

C'est hier matin, quand il m'a demandé de vous écrire à ce sujet qu'il m’a avoué sa véritable pensée. Tout se résume à ceci: "Taqanarmat», c'est fatiguant, je suis fatigué (que je traduis moi-même comme ceci: je suis fatigué de la vie religieuse; je ne lui ai pas demandé directement si, au fond, c'était pour pouvoir se marier qu'il pense à se retirer). Les frères qui l'ont connu ici, il y a trois ans, trouvaient cet été qu'il avait l'air moins heureux qu'autrefois. Il dit lui-même qu'il a cette pensée de sortir depuis un an ou un et demi, mais jusqu'ici n'avait parlé à personne de cette tentation ${ }^{46}$.

Le père Daniélo soupçonne que le célibat est en cause. Une autre piste nous est fournie par le contenu d'une lettre au père Thibert, et dans laquelle Manernaluk affirme qu'il se sentait très préoccupé par la chasse et que tout le reste le fatiguait beaucoup. Lui disant sa peine et sa sympathie, le père Daniélo tente une dernière fois de le convaincre de rester dans les ordres en évoquant sa relation avec le père Buliard:

Je lui ai dit... que je croyais personnellement à la réalité de sa vocation; et de bien réfléchir avant de se décider, de n'envisager que le bien de son âme, pour ne pas connaître d'amers regrets plus tard et à l'heure de sa mort. À mon avis personnel, quoique faillible, c'est une tentation. Mais réfléchis bien devant le

45. AD, HH4230 A62C 19, Lettre du père E. Daniélo au père R. Haramburu, 31 octobre 1962.

46. AD, HH4230 A62C 19, Lettre du père E. Daniélo au père R. Haramburu, 31 octobre 1962. 
Bon Dieu et devant ta conscience... Je lui ai demandé aussi pourquoi il n'en a pas parlé lors de votre séjour récent ici. Pas de réponse claire là-dessus. «Et si tu quittes, où vas-tu aller?» «- Je ne sais pas». Enfin, je lui ai dit: «C'est l'anniversaire de la mort du père Joseph Buliard avec qui tu as mangé de la misère à Garry Lake. Réfléchis aux conseils que, selon toi, il te donnerait luimême s'il était à ma place. Et prions-le ensemble, dans une neuvaine, de nous obtenir la lumière. $»^{47}$.

Les pressions de Daniélo pendant plus de deux jours n'y font cependant rien, pas plus que les bons mots du père Beauregard qui avait souligné le comportement exemplaire de cette jeune recrue au noviciat - sans doute «le meilleur de tous les novices qu'il avait connus là-bas ${ }^{48}$ ». Manernaluk signe sa démission et l'apporte au père Daniélo qui rédige alors un mot à l'évêque, $\mathrm{M}^{\mathrm{gr}}$ Lacroix, le 3 novembre. Il souligne combien Manernaluk souffre à présent de fatigue et de migraines, précisant qu'il n’a à aucun moment transgressé son vœu de chasteté ${ }^{49}$. Daniélo fait part de sa grande peine au père Haramburu, craignant, du coup, de devoir s'inquiéter aussi maintenant pour le frère de Manernaluk, Nick Sikkuaq.

En tout cas cette nouvelle m'est arrivée comme un coup de massue. Nul doute que ce sera une grosse perte pour vous et $\mathrm{M}^{\mathrm{gr}}$ Lacroix, auquel j'envoie le double de cette lettre [...]. Et je crains beaucoup l'influence que cette sortie d'Anthony aura sur son frère Nicolas qui, jusqu'à la décision finale, ne connaitra rien de la chose ${ }^{50}$.

Les archives oblates ne livrent aucune information ni sur la suite des événements ni sur les raisons réelles qui ont conduit Manernaluk à renoncer à sa vocation religieuse. Le Codex historicus de la mission Chesterfield Inlet indique qu'il perd son fils de dix ans dans l'incendie de sa maison en septembre $1975^{51}$. Lors d'une entrevue avec lui à Rankin Inlet en 2006, Manernaluk ne souhaita pas donner plus de détails, faisant valoir son plaisir à fabriquer aujourd'hui des tambours et des couteaux qu'il vend aux touristes de passage, mais surtout à continuer de chasser et de circuler dans la toundra.

Dans une lettre adressée au père Daniélo et datée du 6 novembre 1962, le père Haramburu s'en prend à ce qu'il nomme "un manque d'ouverture» de Manernaluk:

47. AD, HH4230 A62C 19, Lettre du père E. Daniélo...

48. AD, HH4230 A62C 19, Lettre du père E. Daniélo...

49. AD, HH4230 A62C 15, Lettre du père E. Daniélo...

50. AD, HH4230 A62C 19, Lettre du père E. Daniélo...

51. AD, LCB1-200, Codex historicus de Chesterfield Inlet (1971-1992), 2 septembre 1975. 
J'ai mis du temps à écrire cette lettre, mais j'ai cru devoir lui expliquer le plus clairement possible le mécanisme de la vocation. C'est peut-être un peu dur à suivre pour lui, mais vous pourrez reprendre doucement tout cela, en insistant sur ce point que pour lui, il n'a qu'une seule question à répondre: Dieu l'appelle-t-il, oui ou non? Le reste, ça nous regarde et c'est à nous à prendre nos responsabilités, connaissant les circonstances. En répondant, d'après la lettre même qu'il a écrite aux questions que je pose, j'en arrive à la même conclusion que vous: je crois qu'il a la vocation, puisqu'il se sent heureux comme frère, "quand je ne serai plus frère, je le regretterai beaucoup, et même maintenant rien que d'y penser cela me fait beaucoup de peine». Mais il faut que ce soit lui qui prenne la décision et cette responsabilité. Quand je pense à ce cas, j'essaie de voir où a été la faille dans cette vocation, et je crois que c'est dans son manque d'ouverture. Il avoue lui-même qu'il y a plus d'un an qu'il pense à tout cela et les raisons qu'il donne, si elles découvrent sa délicatesse, ne sont pas pour autant des raisons sérieuses. S'il s'était ouvert de ses pensées dès le début, il n’aurait pas été difficile de l’aider à s'en débarrasser, mais je crains que le temps n'ait développé en lui une psychose contre laquelle les raisonnements ne pourront rien. Nous nous heurtons là à une grosse difficulté des tempéraments primitifs: le manque d'ouverture par le manque de connaissance de soi-même et d'expression ${ }^{52}$.

Les Oblats tergiversent. Ils doivent pourtant se résigner, admettre que Manernaluk s'est lassé de la vie religieuse, loin des siens et loin du territoire. Les Oblats ne se remettent pas en cause mais savent qu'ils perdent une vocation et un symbole. Ils ne voient pas que le manque d'ouverture se situe de leur côté, dans le poids d'une culture ecclésiale, et dans une socialité très éloignée de celle qui prévaut dans le monde inuit où une personne reste attachée à des réseaux d'affins. Finalement, dans une lettre du 16 novembre 1962, le père Haramburu accepte que Manernaluk mette fin à sa vocation religieuse. Le père confie éprouver malgré tout un sentiment de fierté devant «tout le beau travail qui s'est accompli dans cette âme » et se demande comment les Oblats pourraient aider Manernaluk à poursuivre sa vie dans le monde laïque, soit en le rattachant à une mission, soit en l'aidant à terminer son cours de mécanique, le but étant de le rendre «le plus indépendant possible ${ }^{53}$ »

De son côté, Anthony Manernaluk avoue qu’il était nerveusement épuisé. En ce sens, sa réaction après quelques années passées auprès des missionnaires oblats ressemble à celle de la sœur Pélagie Inuk qui a également invoqué une lourde fatigue pour quitter l'habit et la congrégation des Sœurs

52. AD, HH4230 A62C 16, Lettre du père R. Haramburu au père E. Daniélo, 6 novembre 1962.

53. AD, HH4230 A62C 17, Lettre du père R. Haramburu au père E. Daniélo, 16 novembre 1962. 
grises $^{54}$. Il faut croire que pour des Inuits de cette époque, travailler continuellement en milieu non inuit, avec des Qallunaat (des Blancs) et dans une autre langue, loin des leurs, était une entreprise difficile et exigeante, tant les cultures et les modes de pensées en présence étaient contrastés. La vraie fatigue de Manernaluk se situe donc en bonne partie dans cette tension continuelle qu'il a dû vivre, lorsqu'il a mesuré le fossé qui séparait les valeurs des Inuits de celles des Qallunaat, ici des missionnaires qui évoluaient dans des communautés en dehors du monde.

Si la décision de Manernaluk ne tient pas à la doctrine chrétienne en tant que telle, la question du mariage n'est pas à écarter, car pour les Oblats, le célibat demeurait une exigence non négociable. Lors de notre entretien en 2006, Manernaluk n’a pas souhaité commenter sa décision, se contentant plus simplement de nous faire part de sa satisfaction à avoir vécu sa vie, tant celle de ses jeunes années auprès du père Buliard que celle qu'il a poursuivie après avoir renoncé à sa vocation religieuse, sans oublier ses années de travail comme mineur à Thompson avant de revenir à Rankin Inlet où il s'est établi, marié, et a fondé une famille, avec la naissance de son fils en 1965. Manernaluk nous confia aussi tout son plaisir à participer activement à un projet de préservation de la tradition orale avec le journaliste David Pelly $y^{55}$.

\section{NICK SIKKUAQ}

Selon le Codex historicus de la mission Saint-Paul de Baker Lake que rédige le père Buliard, Nick Sikkuaq a été baptisé le 4 avril 1948 et fait sa première communion le 2 décembre 1952. Contrairement à son frère Manernaluk, il fait explicitement la demande de s'engager dans les ordres ${ }^{56}$.

"Je veux donner toute ma vie à Jésus... je veux aider les autres ${ }^{57}$ ». Dans une lettre écrite en anglais, datée du 16 mars 1961 et envoyée de Gjoa-Haven, Sikkuaq implore le père de l'aider à satisfaire sa demande :

Mon Père,

J'aimerais être un frère oblat de Marie-Immaculée. Je veux donner ma vie à Jésus. Si vous m'acceptez, je serai très heureux. Je suis le jeune frère du frère Anthony. Vous m'avez vu maintes fois à Gjoa-Haven. Père, je vous en prie,

54. F. Laugrand et J. Oosten, "The case of Pelagie Inuk...», p. 157-176.

55. Manernaluk a cosigné un ouvrage avec N. Sikkuark, Louis Anakanerk et David Pelly intitulé "Going home to Kutgajuk». Ce texte a été publié dans la revue Above and Beyond (juillet-août 2000), p. 34-37 (voir aussi <http:/ / davidpelly.com/Going\%20Home\%20to\%20Kutgajuk.pdf>).

56. AD, HH 4895 A62C 6, Lettre de J. M. Cochard au père A. Lizée, 11 avril 1961.

57. AD, HH 4865 A62C 5, Lettre du 16 mars 1961, signée Nicolas Sitkoa. 
écrivez-moi et dites-moi ce que je dois faire. J'ai 18 ans et je ne souhaite pas me marier. Je veux sauver mon âme et aider les autres à sauver la leur. Je ne peux écrire plus.

Bénissez-moi mon Père

Nicolas Sitkoa ${ }^{58}$

Sikkuaq fait immédiatement référence au don de soi et au célibat, jugeant probablement que ces arguments seraient les plus convaincants. Il indique vouloir aider les autres, une disposition qui évoque peut-être aussi la fonction chamanique.

Le 11 avril 1961, le père Cochard rapporte que la demande de Sikkuaq est sur le point d'être acceptée et que ce dernier sera admis au noviciat au titre de frère coadjuteur. Les autorités voient d'un bon œil qu'il puisse rejoindre son frère Manernaluk et le père Henry, directeur de la mission de Gjoa Haven, l'évalue comme "un excellent sujet», ce que valide l'administration du Vicariat de la Baie d'Hudson ${ }^{59}$. Le 11 avril 1961, le père Cochard écrit à son tour au père $\mathrm{A}$. Lizée, le provincial, puis au père Beauregard, le responsable des novices pour la province du Manitoba, pour leur recommander ce candidat qui pourrait arriver en mai 1961, à l'âge de 18 ans, si tout se passe bien ${ }^{60}$. Le père Cochard insiste sur les qualités de ce jeune homme, soulignant qu'il est équilibré dans son jugement, pondéré, intellectuellement et moralement solide, et bien instruit sur le plan de la religion, et tout particulièrement sur la question de l'eucharistie et de la pénitence ${ }^{61}$. Cochard informe aussi l'évêque $\mathrm{M}^{\mathrm{gr}}$ Marc Lacroix. Le 24 avril, le père Henry indique dans sa lettre au père Beauregard qu'il y a là, en effet, un candidat exceptionnel:

Mon Révérend Père,

Voici quelques informations sur le jeune postulant Nicolas Sitkoa qui peuvent vous être utiles: ce jeune homme est né aux abords de King William Island N.W.T. le 21 mai 1943. Il fut baptisé par le défunt Père Joseph Buliard le 4 avril 1948 à Sherman Inlet N.W.T. et confirmé à Gjoa-Haven par Mgr. Lacroix le 17 avril 1960. Il est le vrai frère de Brother Anthony Manernaluk. Il sort d'une excellente famille. Je l'ai eu sous les yeux depuis 1955. À part quelques petits défauts de jeunesse je n'ai absolument rien à lui reprocher. C'est avant tout un observateur. Il apprendra beaucoup au Noviciat par ce qu'il verra, le bon

58. AD, HH-4865-A62-5, Lettre du 16 mars $1961 \ldots$, traduite par l'auteur.

59. AD, HH-4865-A62-6, Lettre du père J. M. Cochard au père A. Lizée, 11 avril 1961.

60. $\mathrm{AD}, \mathrm{HH}$ 4865-A62C-7, Lettre du père J.M. Cochard au père R. Beauregard, 11 avril 1961.

61. AD, HH-4865-A62C-7, Lettre du père J. M. Cochard au père R. Beauregard, 11 avril 1961. 
exemple qu'il observera. Quant aux exhortations vocales n'ayant qu'une faible pratique de l'anglais il n'y comprendra pas grand chose, du moins la première année de son séjour chez vous. Les seules leçons de langue anglaise qu'il a eues ici n’ont duré que quelques mois d'hiver.

Nicolas est très soigneux dans tout ce qu'il fait. Il est un peu lent. C'est un homme de détail. Comme tous ses frères il est bon dessinateur. Mais sa plus grande qualité c'est de préférer la volonté des autres à la sienne. Il s'est toujours fait remarquer par son obéissance prompte et joyeuse. C'est peut-être là la raison qui ici le faisait aimer de tout le monde. Sur une vie aussi simple que la sienne je ne vois pas ce que je pourrais bien ajouter qui aiderait à sa formation religieuse. Avec la haute idée que nous avons cherché à lui inculquer de la vie divine en nous, de la beauté de la donation totale au service de Dieu et des âmes en fuyant de plus en plus le péché je crois Nicolas à la veille de faire un excellent Noviciat. Dieu suppléera à l'impossible. À vous de le façonner comme vous avez si bien façonné son frère. [...]

Inutile de vous dire combien j'aime Brother Anthony comme nous l'appelons ici. Je ne regrette qu'une chose, qu'il ne soit pas prêtre pour évangéliser sa race. Nous avons tant besoin de prêtres esquimaux pour les Esquimaux. En ce moment nous avons une vocation au sacerdoce en grade 4 à Inuvik, Louis Kringartor. Je rêve de vous l'envoyer dans 5-6 ans ${ }^{62}$.

Sur la base d'un tel appui, l'évêque Lacroix accepte «de tout cœur» l'entrée de Nicolas au noviciat ${ }^{63}$. Sikkuaq commence son postulat avec le père Henry à Gjoa Haven. Le 7 mai, 1961, il arrive à Saint-Norbert, au Manitoba, où il prend l'habit de la main même du père Henry le 21 mai $^{64}$. Sikkuaq y commence son noviciat sous la responsabilité des pères Beauregard, Moissac, Daniélo et Cochard. Il prononce finalement ses premiers vœux le 21 mai 1962 en la présence de son frère, Anthony Manernaluk ${ }^{65}$.

En 1962, les Oblats, qui ont déjà recruté la sœur Pélagie Inuk, se montrent extrêmement fiers de présenter deux vocations masculines. Dans un numéro de La Vie Indienne publié en septembre 1962, le père Gérald Labossière donne un titre bien choisi à son article: "Doublement frères". L'auteur présente les deux hommes et leur projet de devenir prêtres ${ }^{66}$. En juin 1962, un autre article, publié cette fois dans La liberté et le patriote et daté du 8 juin 1962, annonce: "Un deuxième Esquimau canadien présente ses voux à St Norbert». L'auteur évoque "une aurore boréale religieuse »,

62. $\mathrm{AD}, \mathrm{HH}-4865-\mathrm{A} 62-9$.

63. AD, HH-4865C-A62-8, Lettre de Mgr Lacroix au père Beauregard, 15 avril 1961.

64. C. Choque, Joseph Buliard..., p. 222.

65. AD, HH-4865C-A62C-1, Nicholas Sikkuark et HH-4865-A62C-1a.

66. $\mathrm{AD}, \mathrm{HH} 4865 \mathrm{C} \mathrm{A62C} 2$. 
et place ces succès en lien direct avec les succès que les Oblats remportent aussi au Groenland, avec la nomination du père Fynn Lynge, et en Alaska, avec la nomination des premiers diacres par les jésuites.

\section{La personnalité de Nick Sikkuaq}

Un questionnaire réalisé par le père Pierre de Moissac et conservé dans les archives décrit quelques aspects de la personnalité de Sikkuaq et nous renseigne sur les informations et les qualités que recherchaient les Oblats pour leurs recrues.

\section{Réponses}

1. Bonne santé, pas de grandes maladies antérieures, la famille semble jouir d'une bonne santé.

2. Nil.

3. Poli, propre, ordonné.

4. Petit de taille, assez robuste. C'est le vrai type esquimau. Fait pour un climat froid.

5. Calme... plutôt froid. Comme la plupart des esquimaux s'extériorise peu. Énergique, doux, droit. Un grand sens d'observation.

6. Intelligence dans la bonne moyenne. Jugement pratique bon. Habile et même artiste. Études primaires faibles, faute d'école à King-William Island avant 1962. Veut apprendre, aime à se renseigner et pose des questions quand il peut parler l'esquimau. Il parle bien l'esquimau, très peu l'Anglais. Talent pour le chant, le dessin, la peinture.

7. Il n'a pas de métier proprement dit. Il travaille à toutes sortes de besognes: menuiserie, peinture, plomberie etc. Il est habile et appliqué à son travail. Jusqu'ici il a été plutôt dirigé. Semble avoir de la difficulté à apprendre l'Anglais dû au fait qu'il n'est pas allé à l'école étant jeune.

8. Qualités morales et vertus surnaturelles.

A) Esprit de foi, pieux, dévoué, détaché, régulier.

B) Docile, soumis, ouvert envers les supérieurs malgré la difficulté de s'exprimer en Anglais.

C) Sociable, charitable, un bon esprit de communauté.

D) Attaché à sa vocation. Peu de connaissance de l'histoire de la Congrégation à cause des limites imposées par le langage. 
E) Vie spirituelle bonne. Fait des efforts et progresse lentement mais surement.

9. Il voudrait demeurer au Noviciat pour compléter sa formation spirituelle et apprendre quelques métiers. Il veut retourner dans son Vicariat. Il rendra de grands services.

10. L'impression générale est très bonne. On a vraiment rien à lui reprocher. Il a fait de grands progrès sur toute la ligne malgré les difficultés de communication. Je le recommande à votre bienveillance ${ }^{67}$.

Les pères remarquent les grandes qualités manuelles et artistiques de Nick Sikkuaq. Nous y reviendrons. Sa demande est acceptée et Sikkuaq signe la formule ad hoc, «J'atteste que je sens vraiment l'appel de Dieu à l'état religieux et clérical. J'affirme pareillement que j'ai l'intention et le désir de demeurer dans la Congrégation, et d'observer le plus fidèlement toutes les obligations et tous les devoirs de l'état religieux ${ }^{68}$.»

Le 30 mars 1964, une seconde évaluation de Nick Sikkuaq est menée par le Saint Rosaire du Scolasticat, au moment où l'Inuk doit prononcer ses troisièmes vœux le 21 mai 1964 :

Bon à tout moment. Il est charitable et prêt à aider les autres. Il est fidèle et fiable pour demander des autorisations et obéissant aux directives qui lui sont données. Il participe très régulièrement aux exercices religieux de la communauté. Son esprit de foi semble être sincère. Sa vie spirituelle en général, d'après ce qu'on peut en observer, semble assez satisfaisante. C'est un très bon travailleur dévoué à son travail. Il fait des progrès dans l'apprentissage de la langue anglaise. Frère Sikkuark donne l'impression d'être un religieux très obligeant, régulier et bon. De ce que nous avons pu observer pendant une période de six mois, il ne présente aucun problème pour avancer et prononcer ses troisièmes vœux temporaires ${ }^{69}$.

Mais un autre document daté du 4 avril 1965 soulève un problème de langue:

La communication sur le plan spirituel avec frère Nicolas est pratiquement impossible à cause de sa connaissance limitée de l'anglais et de notre ignorance complète de l'Esquimau. La direction et la décision sur sa vie spirituelle

67. $\mathrm{AD}, \mathrm{HH}-4865-\mathrm{A} 62 \mathrm{C}-13$.

68. AD, HH-4865-A62C-14, Novice's Personal request to make his profession, April 10, 1962.

69. AD, HH-4865-A62C-19 ex1, Temporary vows, Missionary Oblates of Mary Immaculate. St. Peter's Province, March 30, 1964. 
intérieure sont entre les mains de ces pères Oblats qui peuvent communiquer avec lui en Esquimau ${ }^{70}$.

La situation semble exceptionnelle, mais les Oblats s'en remettent à leurs confrères du Nord qui parlent la langue des Inuits.

La suite du rapport de Nick Sikkuaq est très positive à l'exception de sa santé. Les Oblats reconnaissent que Sikkuaq est plein de dévotion, qu'il fait bien ses exercices spirituels, mais «sa maladie limite la quantité de travail qu'on peut lui assigner. Il se fatigue facilement.» Malgré tout, le conseil recommande qu'il formule maintenant ses troisièmes vœux ${ }^{71}$.

Avec la bénédiction du Conseil provincial des Oblats, le frère Sikkuaq exprime son choix de poursuivre sa formation et sa vie religieuses et le Conseil vote à l'unanimité son admission à franchir l'étape de ses troisièmes vœux, le 21 mai 1965. Comparativement à son frère aîné, Sikkuaq franchit donc une étape de plus. Le père R. Haramburu suggère alors de l'envoyer à Ottawa afin d'y être supervisé par le père Daniélo qui venait lui aussi d'arriver là en raison de problèmes de santé ${ }^{72}$.

Mais le 19 mai 1964, Nick Sikkuaq fait une rechute et il est envoyé à l'Hôpital général d'Ottawa. Une lettre du père McGrath à l'hôpital du Manitoba à Winnipeg, le 20 mai 1964, suggère que Sikkuaq souffrait en effet de sérieux problèmes de santé.

Après cet événement, les archives ne livrent malheureusement plus d'information et l'on perd la trace du frère Sikkuaq. On sait toutefois que l'Inuk ne voulut pas rester dans la congrégation et qu'il retourna dans l'Arctique où il devint un très grand sculpteur, un peintre et un dessinateur, avant de décéder en 2013.

\section{Frère et artiste passionné par les petites bestioles (qupirruit) et le chamanisme}

Pendant sa formation religieuse, les Oblats avaient déjà perçu les talents artistiques et la créativité de Nick Sikkuaq. En 1973, d'ailleurs, à la demande du Keewatin Region Education Office and Department of Education of the NWT, Sikkuaq publia quatre livrets dans lesquels il signe de fascinants dessins avec leurs récits. Intitulé Faces le premier volume présente une série de magnifiques portraits. Le second, What animals think est tout aussi réussi avec de nombreuses réflexions que l'Inuk attribue aux animaux. Le troisième ouvrage est presque surréaliste et évoque le célèbre

70. AD, HH-4865-A62C-20, Nicholas Sikkuark, April 4/May 21, 1965.

71. AD, HH-4865-A62C-20, Nicholas Sikkuark..

72. AD, HH-4865-A62C-23, Letter from R. Haramburu to Father McGrath, April 23, 1964. 
catalogue des objets introuvables. Intitulé Nick Sikkuark's Book of things you will never see, l'ouvrage met en valeur sa créativité. Quant à son dernier livre, More stories (1973), il illustre encore son imagination ${ }^{73}$.

Sikkuaq ne fut toutefois pas un artiste comme les autres. Son œuvre est marquée par plusieurs thèmes singuliers, comme celui des petites bestioles (qupirruit) qu'il a traité, en effet, de mille et une manières et qui apparait dans son œuvre, comme une véritable obsession. Les qupirruit constituent un thème chamanique par excellence ${ }^{74}$, ce qui laisse entendre que l'artiste a toujours conservé cet intérêt pour le rôle des esprits. Plusieurs de ses qupirruit apparaissent dans un livre édité par Robert Kardosh ${ }^{75}$ dans lequel il les fait intervenir comme des entités puissantes et agressives. D’autres sont associées à des figures ancestrales capables de se déplacer très vite et représentées sous la forme de vers pourvus de têtes humaines. Sikkuaq n'était pourtant pas un chamane initié mais ses œuvres montrent qu'il était qualifié pour le devenir. Il est possible que son intérêt pour le chamanisme demeure à la base de son souhait de devenir prêtre pour aider les autres. Il est vrai qu'aux yeux de nombreux observateurs et anthropologues, les missionnaires avaient pris le rôle des chamanes et pouvaient bien être perçus comme tels. Nul autre que $\mathrm{M}^{\mathrm{gr}} \mathrm{A}$. Turquetil le dira : "Ces pauvres gens nous prennent pour des $\operatorname{sorciers}^{76}$."

\section{Discussion}

Avant les années 1950, les Oblats ne voulaient pas former un clergé indigène. Mais les premières vocations, notamment celle de sœur Pélagie, ouvraient une nouvelle ère et laissaient espérer d'autres recrues. Au milieu des années 1960, cependant, alors que le concile Vatican II ouvre de nouvelles perspectives dans ce domaine, leurs ambitions s'écroulent. Leurs efforts en matière de recrutement et de formation d'un clergé indigène se heurtent à des barrières infranchissables.

Dans le cas de Manernaluk qui s'est marié, les Oblats se doutent que le célibat a joué un rôle dans sa défection, quand bien même les archives ne disent pas un mot de cette frustration. Manernaluk indique son découragement mais à son retour dans le nord, il se marie finalement avec une

73. Ces livres difficiles à trouver ont été publiés en 1973 à Yellowknife par le Curriculum Division du Département d'éducation du Gouvernement des Territoires du Nord-Ouest.

74. Frédéric Laugrand et Jarich Oosten, «Maîtres de la vie et de la mort. La grandeur des "petites bêtes” du Grand Nord». L'Homme. Revue française d'anthropologie, 202 (2012), p. 53-76.

75. Robert Kardosh, The Art of Nick Sikkuark: Sculpture and Drawings (Vancouver, Marion Scott Gallery, 2003), p. 84-85.

76. F. Laugrand, Mourir et renaître..., p. 148. 
jeune veuve de Garry Lake, Lucie Tapvi. Pélagie fera de même en rejoignant le mari qui lui avait été promis jadis.

Dans les années 1970, le père R. Lechat, un missionnaire oblat qui a longtemps séjourné au Nunavik, puis au Nunavut, s'exprimera ouvertement sur ce sujet. Proposant d'abolir cette exigence, le missionnaire déclenchera une vaste polémique au sein de son diocèse et jusqu'à Rome ${ }^{77}$. La situation paraît plus floue dans le cas de Nick Sikkuaq, atteint par la maladie.

Quoiqu'il en soit, ces deux frères ne sont pas allés au terme de leur vocation religieuse en raison de l'abîme qui, à leurs yeux, les séparait de la culture des Blancs. Ce fossé a alimenté la maladie et généré une forme d'épuisement psychologique. Aujourd'hui, en effet, on sait que les Inuits n'aiment pas vivre seuls et loin des leurs trop longtemps, et combien les valeurs familiales restent importantes. Les Oblats n'ont rien fait pour diminuer ce fossé culturel, prisonniers eux-mêmes d'une institution aux règles rigides. Sur le terrain, les missionnaires mesuraient pourtant bien les différences. Bien avant Vatican II, le père Turquetil s'était battu pour obtenir la reconnaissance du privilège paulin et des dérogations du droit canonique sous la forme de "dispenses» pour le mariage des impubères ${ }^{78}$. Mais dans les établissements du Sud, les Oblats ne disposaient pas d'une marge de manœuvre suffisante pour adapter leur formation aux rares vocations indigènes. Eu égard aux Inuits, il faudra attendre les années 1970 pour que les Oblats trouvent des solutions, optant finalement pour la formation de couples catéchistes.

À ce jour, aucun Inuit n'est devenu prêtre. Sachant que les Anglicans ont pour leur part su recruter de nombreux leaders, mariés pour la plupart, comment ne pas admettre que le célibat demeure un obstacle majeur? Louis Tapardjuk, ancien organiste de la communauté catholique d'Igloolik dans les années 1960, s'est exprimé sur ce point:

Les Inuits n'ont pas l'habitude d'être seuls. Ce n'est pas leur coutume de ne pas avoir de famille. La famille vient en premier. Vous avez besoin d'une femme pour s'occuper de vos besoins, comme pour la confection de vos kamiit (bottes) et tout le reste. Souvent, les ministres anglicans avaient des familles, mais jamais les prêtres catholiques romains. Il n'y a aucune chance qu'un Inuk

77. R. Lechat, «L'église de demain ». Conférence à la réunion conjointe des missionnaires des diocèses de Churchill Baie d'Hudson et Mackenzie tenue à Fort Smith en 1974. Manuscrit inédit communiqué par l'auteur.

78. Arsène Turquetil, «Le mariage chez les Esquimaux en regard des facultés de dispense accordées aux missionnaires ", Revue de l'Université d'Ottawa, 5, 4 (1935), p. 125-137. 
puisse devenir prêtre à moins que le Vatican ne change ses règles et permette aux prêtres de se marier ${ }^{79}$.

En conséquence, l'Église catholique est toujours restée dépendante de non-Inuits pour recruter.

Dans le cas de Manernaluk et de Sikkuaq, il est peu probable que le célibat suffise à lui seul à expliquer l'échec de leur vocation. En effet, de grandes similitudes existent si l'on compare les problèmes qu'ont expérimentés la sœur Pélagie et les deux frères. Pélagie et Sikkuaq ont évoqué des problèmes de langue, de sorte que la communication avec leurs camarades et les autres membres de la congrégation a toujours été difficile. En outre, ils ont opté pour une vie solitaire tout en devenant membres d'un ordre qui avaient ses propres traditions, son style et ses règlements, or ces institutions n'avaient rien à voir avec les traditions inuites où la vie en communauté ouverte prime. Le père Henry avait raison de souligner l'existence de blocages d'ordre culturel: «l'abîme profond qui sépare les mentalités blanches et inuit, les différentes habitudes alimentaires, les coutumes et le climat; la barrière de la langue créant des difficultés dans la communication, le découragement ${ }^{80}$ ». La structure formelle et la hiérarchie stricte des ordres religieux contrastaient nettement avec l'organisation plutôt égalitaire de la société inuite. La plupart du temps, Pélagie, Manernaluk et Sikkuaq étaient les seuls Inuits présents dans la congrégation, ce qui devait renforcer leur sentiment de solitude et d'isolement. Les règles du comportement et l'expression des sentiments étaient acceptables pour des Blancs, mais cette discipline a dû peser très fortement sur les Inuits. Parions que ces éléments sont à la source de cette fatigue et des migraines que rapportent les deux frères comme la sœur Pélagie.

Dans tous les cas, ces défections découlent probablement moins d'un manque de foi - ces Inuits resteront d'ailleurs longtemps au service du diocèse après leur départ -, que d'un refus de changer radicalement de vie sociale. Ces facteurs, et l'obligation de fonctionner dans un contexte exogène, ont épuisé ces jeunes Inuits qui n’ont pu résister indéfiniment à cette désocialisation que la christianisation et la modernisation assimilatrice engendraient. Leur vie sociale a été perturbée et même la compassion de leurs confrères oblats n'a pu compenser ces manques. Manernaluk et Sikkuaq ont ainsi préféré retourner dans le Nord et fonder une famille,

79. Louis Tapardjuk, Fighting for our Rights (Iqaluit, Nunavut Arctic College, 2013), p. 46.

80. C. Choque, Joseph Buliard..., p. 205. 
heureux de retrouver là une vie sociale plus intégrée et un mode de vie plus convenable à leur idéal.

Les missionnaires ont souvent pensé que le chamanisme et le christianisme étaient incompatibles et ont donc tout fait pour supprimer les pratiques chamaniques, les chamanes étant considérés comme des "charlatans» et les interdits rituels comme des «superstitions ${ }^{81}$ ». Cependant, le chamanisme n'a pas disparu. Il est devenu invisible, nombre de pratiques ayant continué à se transmettre dans les camps, à l'écart des missionnaires ${ }^{82}$. Alors que les Oblats et les Sœurs grises ne recourraient évidemment jamais au chamanisme, les Inuits catholiques pouvaient à l'occasion intégrer les pratiques chamaniques dans une perspective chrétienne, une dynamique qui s'observe avec la confession par exemple. La logique du chamanisme pouvait ainsi encore fonctionner dans un contexte chrétien, comme l'illustre encore le don que la sœur Pélagie a fait au chamane Suluk, en accrochant un objet à sa ceinture pour que ce dernier puisse l'aider à devenir une sœur grise ${ }^{83}$. Dans ses œuvres, l'artiste Nick Sikkuaq laisse apparaitre sa passion pour le chamanisme mais uniquement comme source d'inspiration artistique. Sur le plan des pratiques religieuses, en effet, l'Inuk avait semble-t-il choisi le christianisme. À cet égard, il se pourrait donc bien qu'en quittant les ordres et en retournant à un mode de vie et de pensée proprement inuit, ces deux frères indiquaient aux Oblats leur volonté de conserver leur identité propre, leur ancrage dans les traditions inuites.

\section{CONCLUSION}

Entre 1912 et le début des années 1960, les pères Oblats et leur évangélisation ont eu un grand impact sur la culture inuite. Ces missionnaires ont réussi à convertir les Inuits au christianisme et, pendant longtemps, ils ont été des autorités incontournables dans les communautés. Mais un élément fondamental que les Oblats n’ont pas réussi à bouleverser est le célibat et, avec lui, les valeurs sociales et relationnelles des Inuits. La parenté inuite demeure d'ailleurs toujours un système fort déconcertant pour les Occidentaux.

Comme la sœur Pélagie, les frères Anthony Manernaluk et Nick Siqquaq étaient des candidats très prometteurs pour les missions catholiques

81. F. Laugrand, Mourir et renaitre..., p. 344.

82. Voir Cornelius Remie, "Culture Change and Religious Continuity Among the Arviligjuarmiut of Pelly Bay, NWT, 1935-1963», Études Inuit Studies, 7, 2 (1983), p. 53-77.

83. F. Laugrand et J. Oosten, Transitions and Transformations... 
et l'indigénisation du christianisme. Tous sont toutefois restés tiraillés entre les valeurs traditionnelles de la vie inuite d'une part, et celles de la religion chrétienne et de l'idéologie des Qallunaat, d'autre part. À la longue, ces Inuits ont rejeté la vie célibataire qu'impliquait leur vocation sacerdotale pour retourner à la vie familiale inuite. Il n'est pas certain que les Oblats aient saisi la force de ce verrou même si, un peu plus tard, ils s'ouvriront davantage aux traditions inuites et à leurs valeurs en choisissant de former des couples catéchistes. Ce blocage comporte enfin une part de paradoxe, dans la mesure où les Oblats ont été des champions de l'adaptation sur le plan social ${ }^{84}$ et, pour certains, des ethnologues avant l'heure ${ }^{85}$.

De manière plus générale, les cas de Manernaluk et de Sikkuaq montrent que l'individualisme moderne, contrairement à une idée admise chez les anthropologues de la religion, n'a pas constitué pour les Inuits l'élément le plus attractif du christianisme, du moins pas à long terme. Dès le départ, les deux frères sont devenus des frères oblats en restant à l'intérieur des réseaux d'affinité qu'ils ont fait fonctionner - Manernaluk comme enfant adopté par le père Buliard et Sikkuaq comme protégé du père Henry. Leur décision de s'engager dans le sacerdoce s'inscrit d'abord dans cette logique.

Plus encore, l'individualisme auquel ils ont été confrontés en commençant leur formation est rapidement devenu un obstacle. De ce point de vue, cette valeur a constitué le blocage principal qui a empêché les deux frères de poursuivre leur vocation religieuse. Si nombre d'idées chrétiennes ne posaient pas problème - le partage, la prière, la confession, la dévotion des saints, etc. -, d'autres exigences comme la sociabilité missionnaire, le célibat et l'isolement du Nord, l'usage du français, l'obéissance et l'idée d'un individu seul face à Dieu, posaient en revanche des difficultés d'un autre ordre en ce qu'elles bouleversaient les valeurs et la socialité inuites.

84. Les Oblats ont accepté très tôt que les Inuits conservent la tradition des mariages arrangés, l'éponymie, la danse au tambour et les festins cérémoniels. Voir Frédéric Laugrand, «Les "déplacements culturels" des missionnaires oblats : une dynamique fondamentale de l'évangélisation des Inuit du Nord canadien ", Études oblates de l'Ouest /Western Oblate Studies, 5 (2000), p. 11-36.

85. Voir Frédéric Laugrand, «La contribution des missionnaires oblats à l'anthropologie des Inuit et des Amérindiens du Canada", dans Pierre Hurtubise, Un passé à découvrir ou redécouvrir/A Past to Be Visited or Revisited. Actes du Premier Symposium consacré à l'histoire des missions oblates auprès des Premières Nations (Ottawa, Université Saint-Paul, Chaire de recherche en histoire religieuse du Canada, 2015), p. 123-169. 
En ce sens, si les Inuits ont adopté le christianisme, ils ont su maintenir des différences et une autre sensibilité, refusant bel et bien la désocialisation radicale qu'il impliquait. 